\title{
Association analysis of dietary habits with gut microbiota of a native Chinese community
}

\author{
LEIMIN QIAN $^{1,2}$, RENYUAN GAO $^{3,4}$, LEIMING HONG $^{3,4}$, CHENG PAN $^{3,4}$, \\ $\mathrm{HAO} \mathrm{LI}^{3,4}$, JIANMING HUANG ${ }^{2}$ and HUANLONG QIN ${ }^{1,3,4}$
}

\begin{abstract}
${ }^{1}$ Department of General Surgery, The Affiliated Shanghai No. 10 People's Hospital of Nanjing Medical University, Shanghai 200072; ${ }^{2}$ Department of Gastrointestinal Surgery, Jiangyin People's Hospital, Jiangyin, Jiangsu 214400;

${ }^{3}$ The Tenth People's Hospital Affiliated to Tongji University, Shanghai 200072; ${ }^{4}$ Research Institute of

Intestinal Diseases, School of Medicine Tongji University, Shanghai 200092, P.R. China
\end{abstract}

Received November 20, 2017; Accepted May 22, 2018

DOI: $10.3892 /$ etm.2018.6249

\begin{abstract}
Environmental exposure, including a high-fat diet (HFD), contributes to the high prevalence of colorectal cancer by changing the composition of the intestinal microbiota. However, data examining the interaction between dietary habits and intestinal microbiota of the Chinese population is sparse. We assessed dietary habits using a food frequency questionnaire (FFQ) in native Chinese community volunteers. Based on the dietary fat content determined using the FFQ, the volunteers were divided into HFD group $(\geq 40 \%$ of dietary calories came from fat) or low-fat diet (LFD) group $(<40 \%)$. Fecal and colonic mucosal microbiota composition was determined using $16 \mathrm{~S}$ rDNA based methods. In stool matter of HFD group, Prevotella and Abiotrophia showed significantly higher abundance, whereas unclassified genus of S24-7 (family level) of Bacteroidetes, Gemmiger, Akkermansia and Rothia were
\end{abstract}

Correspondence to: Dr Huanlong Qin, Department of General Surgery, The Affiliated Shanghai No. 10 People's Hospital of Nanjing Medical University, 301 Yanchang Middle Road, Zhabei, Shanghai 200072, P.R. China

E-mail: huanlong_qin@live.cn

Abbreviations: CRC, colorectal cancer; IBS, irritable bowel syndrome; IBD, inflammatory bowel diseases; CDAD, Clostridium difficile associated diarrhea; LPS, lipopolysaccharide; GI, gastrointestinal; HFD, high-fat diet; HCAs, heterocyclic amines; BMI, body mass index; NSAID, non-steroidal anti-inflammatory drugs; HIV, human immunodeficiency virus; LFD, low-fat diet; FFQ, food frequency questionnaire; DNA, deoxyribonucleic acid; PCR, polymerase chain reaction; RNA, ribonucleic acid; PEs, paired-end reads; OTUs, operational taxonomic units; RDP, ribosomal database project; SDI, Shannon diversity index; LDA, linear discriminant analysis; SPSS, statistical package for social sciences; SEM, standard error of the mean; PCoA, principal coordinate analysis; SCFA, short-chain fatty acids

Key words: high-fat diet, gut microbiota, colorectal diseases, feces, colonoscopy less abundant. On colonic mucosal tissue testing, unclassified genus of S24-7 showed significantly higher abundance while Bacteroides, Coprobacter, Abiotrophia, and Asteroleplasma were less abundant in HFD group. A high fat and low fiber diet in a native Chinese community may partially contribute to changes of intestinal microbiota composition that may potentially favor the onset and progression of gastrointestinal disorders including inflammatory, hyperplastic and neoplastic diseases.

\section{Introduction}

Colorectal cancer (CRC) is the second leading cause of gastrointestinal tumors with an estimated 380,000 newly diagnosed cases in China in 2011 (1). In addition to genetics, environmental factors, such as lifestyle and dietary habits, are associated with CRC development $(2,3)$. Colonic epithelium is exposed to digested food substances, which may initiate cellular transformation and tumor progression through a variety of direct or indirect processes. It has been suggested that in these processes, the intestinal microbiota plays an important role. With the aid of genetic $16 \mathrm{~S}$ ribosomal RNA polymerase technique, intestinal dysbiosis has been detected in CRC-associated gut diseases, such as irritable bowel syndrome (IBS) $(4,5)$, inflammatory bowel diseases (IBD) $(6-8)$ and Clostridium difficile associated diarrhea (CDAD) (9). However, it is interesting to note that most of the studies on intestinal micro biota have been conducted on CRC patients with sparse knowledge regarding microbiota in healthy human populations (10).

Dietary fat is the third major source of energy in humans. Ingested fat is conjugated to bile acids, which transform fat into bioactive metabolites absorbed by the intestinal epithelial cells. Primary bile acids that escape intestinal absorption undergo microbe-mediated enzymatic deconjugation in the terminal ileum or colon and hence produce secondary bile acids, which are potentially carcinogenic and have been implicated in the etiology of CRC and other GI diseases $(11,12)$. Key microbiota responsible for deconjugation of primary bile acids from taurine and glycine have been identified as Bifidobacterium, Lactobacillus and Bacteroides (13). Numerous lipids with 
biological activity are also produced by bacteria, including lipopolysaccharide (LPS), a component of the cell wall of gram negative bacteria, which can result in tissue inflammation in the gut and other organs, chronic low grade systemic inflammation in particular, and initiate the development of many metabolic diseases. Consequently, dietary fat can influence the composition and metabolic activity of the gut microbiota and an imbalance in microbiota can adversely impact the digestion and absorption of fat.

Consumption of a high-fat, low-fiber diet has been shown to be associated with various pathologies, including obesity, diabetes, hypertension, cardio-cerebrovascular disease and cancer. A high-fat diet (HFD) affects the colonic environment by increasing exposure to mutagens such as heterocyclic amines (HCAs) formed during the cooking and processing of meat $(14,15)$, and by affecting the composition and diversity of gut microbiota in CRC populations $(2,16-18)$. Two studies in inbred mice and human have shown that the shift of dietary macronutrients could significantly alter the gut microbiota within a single day $(19,20)$. However, it remains unclear how the gut microbiota respond to a HFD and low-fat diet (LFD) in healthy individuals. In this study, we investigated the changes of gut microbiota composition upon the differences in the fat content of the diet in a Chinese community.

\section{Patients and methods}

Target population. We enrolled volunteers in the age group of 45-65 years (high risk group for cancer as per World Health Organization guidelines) (21) with a BMI of $18.5-30 \mathrm{~kg} / \mathrm{m}^{2}$, and able to provide written consent and information regarding basic demographic data as well as dietary history. We excluded subjects who had congenital intestinal defects and or previous gastrointestinal operation(s); chronic gastrointestinal diseases such as inflammatory bowel disease; acute gastrointestinal diseases including acute gastroenteritis and/or ulcers; a prior diagnosis of any cancers; history of hospitalization or use of systemic antibiotics, lipid-lowering agent, probiotics and prebiotics within the past six weeks and non-steroidal anti-inflammatory drugs (NSAIDs) within the past four weeks; and HIV infection. This study was approved by the Institutional Review Boards of Shanghai Tenth People's Hospital (Shanghai, China) and Jiangyin People's Hospital (Jiangyin, China).

Ultimately, twenty-nine volunteers were approached to participate in the study through local community health service center. After enrollment, each volunteer received a study kit including all the questionnaires and a sterile stool collection kit. This study used the self-administered Block 95 food frequency questionnaire (FFQ) to assess the dietary habits for the last one year of enrolled subjects $(22,23)$. The amount of fat, carbohydrate, proteins and dietary fiber in various food products consumed daily were analyzed using the criteria of China Food Composition (Book 1.2nd Edition) and the energy yield of the first three nutrients was derived (9, 4 and $4 \mathrm{kcal} / \mathrm{g}$, respectively). Based on the dietary fat content determined using the FFQ, the volunteers were divided into HFD group ( $\geq 40 \%$ of dietary calories came from fat) or LFD group $(<40 \%)$. Demographic information, familial medical history (e.g., familial gastrointestinal tumors, hypertension and diabetes) and other lifestyles including smoking, alcohol consumption, intensity of physical labor and exercise, were assessed using a scalable questionnaire specifically developed for this study. Physical examination including height, weight, waist circumference and hip circumference were also recorded. The background information of the two groups is shown in Table I.

Stool collection and colonic mucosal tissue sampling. After filling out the questionnaires, a freshly voided stool sample was collected from volunteers. Out of 14 volunteers in LFD group, 3 subjects have no stool specimens but perform biopsies of intestinal mucosa which are used for analysis of mucosa-associated microbiota. Finally, 15 stool samples from HFD group and 11 from LFD group were obtained. All sample kits were immediately placed in an incubator containing several ice packs and then transported to the laboratory within two hours. Upon arrival in the laboratory, each sample was immediately homogenized in a number of microtubes and frozen at $-80^{\circ} \mathrm{C}$ waiting for microbiota analysis.

In order to visualize the intestinal mucosa and collect colonic mucosal tissue, colonoscopic examination and biopsy were performed. Before the procedure, the subjects were provided with comprehensive health education including procedural information and related potential hazards, and written informed consent was obtained. Final only 24 subjects (13 and 11 for HFD and LFD groups, respectively) gave consent for biopsy. Colonoscopy data showed higher incidence of abnormalities in HFD group including polyps mainly, diverticula and colitis. Most polyps were found under $0.5 \mathrm{~cm}$ in diameter and pathologically classified as mixed polyps and low-grade intraepithelial neoplasia (data not shown). Biopsies were obtained from the ascending colon with biopsy forceps. The normal colonic tissue samples were placed into sterile microtubes and then promptly transported to a Thermos bottle containing liquid nitrogen. Finally, the tissue samples were frozen $-80^{\circ} \mathrm{C}$ together with stool samples for microbiota analysis.

\section{Microbiota analysis}

DNA extractions. DNA from stool samples and normal colonic mucosal tissue samples, most of which were collected from the same volunteers, was extracted using the Micro Elute Genomic DNA kit (D3096-01; Omega Bio-Tek, Inc., Norcross, GA, USA) according to the manufacturer's instructions. The reagent used to isolate DNA from trace amounts of sample was effective for the preparation of DNA of the majority of bacteria. Sample blanks consisted of unused swabs processed through DNA extraction and were tested to contain no $16 \mathrm{~S}$ amplicons. The total DNA was eluted in $50 \mu \mathrm{l}$ of elution buffer by modification of the procedure described by manufacturer (Qiagen, Hilden, Germany) and stored at $-80^{\circ} \mathrm{C}$ until measurement in the PCR by LC-Bio (Hangzhou, China).

PCR amplification and $16 S$ rDNA sequencing. Using total DNA from the samples as a template and the primer (319 forward, 5'-ACTCCTACGGGAGGCAGCAG-3'; 806 reverse, 5'-GGACTACHVGGGTWTCTAAT-3'), we amplified the $\mathrm{V} 3-\mathrm{V} 4$ region of the bacterial 16S rRNA. All reactions were carried out in $25 \mu \mathrm{l}$ (total volume) mixtures containing approximately $25 \mathrm{ng}$ of genomic DNA extract, $12.5 \mu \mathrm{l}$ PCR Premix, 
$2.5 \mu \mathrm{l}$ of each primer, and PCR-grade water to adjust the volume. PCR reactions were performed in a MasterCycler Gradient Thermal Cycler (Eppendorf, Hamburg, Germany) set to the following conditions: initial denaturation at $98^{\circ} \mathrm{C}$ for $30 \mathrm{sec}$; 35 cycles of denaturation at $98^{\circ} \mathrm{C}$ for $10 \mathrm{sec}$, annealing at $54 / 52^{\circ} \mathrm{C}$ for $30 \mathrm{sec}$, extension at $72^{\circ} \mathrm{C}$ for $45 \mathrm{sec}$; and then final extension at $72^{\circ} \mathrm{C}$ for $10 \mathrm{~min}$. The PCR products were identified using $2 \%$ agarose gel electrophoresis. Throughout the DNA extraction process, ultrapure water, instead of the sample solution, was used to exclude the possibility of false-positive PCR results as a negative control. PCR products were normalized by AxyPrep ${ }^{\mathrm{TM}}$ Mag PCR Normalizer (Axygen Biosciences, Union City, CA, USA), which allowed us to skip the quantification step, regardless of the PCR volume submitted for sequencing. The amplicon pools were prepared for sequencing with AMPure XT beads (Beckman Coulter Genomics, Danvers, MA, USA) and the size and quantity of the amplicon library were assessed on the LabChip GX (Perkin Elmer, Waltham, MA, USA) and with the Library Quantification kit for Illumina (Kapa Biosciences, Woburn, MA, USA), respectively. PhiX Control library (v3) (Illumina, San Diego, CA, USA) was combined with the amplicon library (expected at $30 \%$ ). The library was clustered to a density of $\sim 570 \mathrm{~K} / \mathrm{mm}^{2}$. The libraries were sequenced on 300PE MiSeq runs and one library was sequenced with both protocols using the standard Illumina sequencing primers, eliminating the need for a third or fourth index read.

Bioinformatic analysis. High-quality 300 bp paired-end reads (300 PEs) were overlapped by $\sim 90 \mathrm{bp}$. The assembled sequences were clustered by using the CD-hit-est based clustering method (24). Software PyNAST (http:/qiime.org/ pynast/) was used to analyze and calculate the numbers of sequence and operational taxonomic units (OTUs) for each sample. Subsequently, the species abundance and distribution were analyzed followed by cluster analysis. After cluster analysis, the sequences were grouped into various OTUs using Felsentein-corrected similarity matrices such that the sequences within an OTU share at least 97\% similarity. The Ribosomal Database Project (RDP) classifier was used to classify the $16 \mathrm{~S}$ rDNA into distinct taxonomic category by aligning sequences to a curated database of taxonomically annotated sequences. All 16S rDNA sequences were mapped to the RDP database using BLASTN in order to achieve taxonomic assignments. Sequences $>97 \%$ identity were used to associate a group of OTUs to specific species, while those with $<97 \%$ identity were considered novel reads. The rank abundance curve was made to roughly assess species abundance and species uniformity. The microbial $\alpha$ diversity in individual stool and colonic mucosal tissue samples was estimated using rarefaction analysis, including four indices: Observed_species, Shannon, Simpson and Chaol indexes. We used three different algorithms to calculate the distance matrices between the samples: Euclidean, unweighted_unifrac, and weighted_unifrac. The Unweighted Pair Group Method with Arithmetic Mean (UPGMA) for clustering of samples was also carried out on Euclidean, unweighted_unifrac and weighted_unifrac matrices. Correspondingly, principal coordinates were 

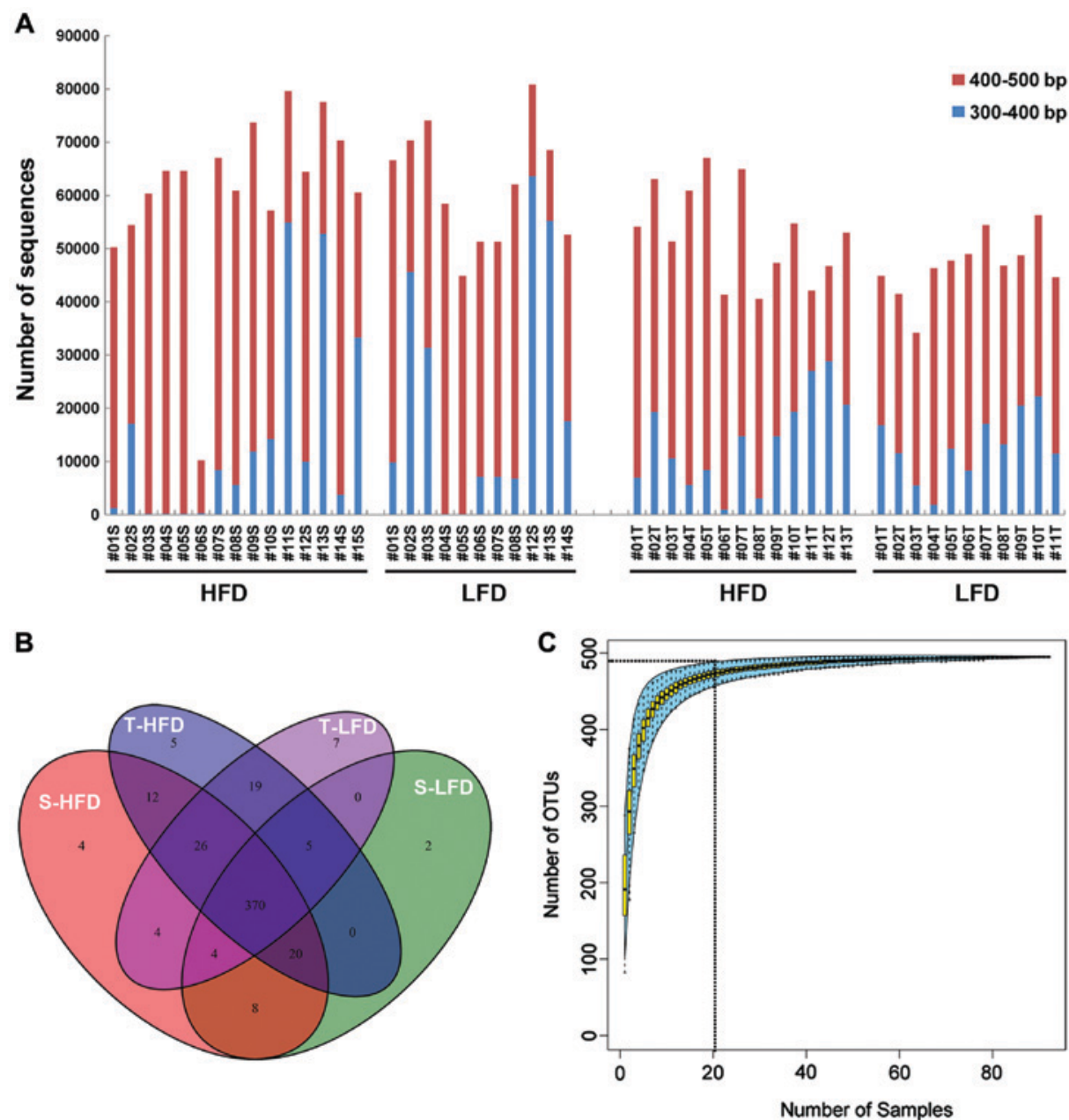

Figure 1. Analysis of sequences and OTU distribution in each of the 26 stool samples and the 24 colonic mucosal tissue samples. (A) The number of sequences at two intervals of 300-400 bp and 400-500 bp in each sample. (B) Venn diagram of OTUs defined at $97 \%$ similarity level. Red indicating stool samples of high-fat diet group (abbreviated as S-HFD); green stool samples of low-fat diet group (S-LFD); blue colon mucosa tissue samples of high-fat diet group (T-HFD) and purple colon mucosa tissue samples of low-fat diet group (T-LFD). (C) Species accumulation curve. OTU, operational taxonomic unit; HDF, high-fat diet; LFD, low-fat diet.

computed for above three distance matrices and used to generate Principal Coordinate Analysis (PCoA) plots. A metagenomic biomarker discovery approach was employed with LEfSe [linear discriminant analysis (LDA) coupled with effect size measurement] which performed a non-parametric Wilcoxon sum-rank test followed by LDA analysis using online software (http://huttenhower.sph.harvard.edu/galaxy/) to assess the effect size of each differentially abundant taxon.

Statistical analysis. Statistical analysis was performed using the Statistical Package for Social Sciences (SPSS) version 19.0 (SPSS, Inc., Chicago, IL, USA). Data were presented as mean \pm standard error of the mean (SEM) for continuous variables, and as percentages for categorical variables. The Student's t-test or Wilcoxon's rank sum test was used to analyze intergroup differences in continuous variables, and categorical variables were compared using Chi-square and ANOVA was used for multiple comparison using Fisher's exact tests as a post hoc test. The relative abundances of stool and tissue bacteria were compared between HFD and LFD groups using the Mann-Whitney and Wilcoxon's tests. The level of significance was set at $\mathrm{P}$-value $<0.05$.

\section{Results}

Comparison of richness and diversity of gut microbiota sequencing. To characterize the pattern of microbiota, we performed $16 \mathrm{~S}$ ribosomal DNA gene sequencing on 50 samples including 26 stool samples and 24 colonic mucosal tissues collected from the HFD and LFD groups. On a whole, 2,799,033 high-quality sequences were generated from usable raw data after optimization process. Eighty-four percent of the sequences were distributed in the interval of 400-500 bp, which corresponded to the V3-V4 region of 16S rDNA gene (Fig. 1A). After CD-HIT clustering, the numbers of OTUs from all samples at $97 \%$ similarity level was 486 . The Venn diagram shows the similarity of OTU distribution (Fig. 1B). The same OTUs accounted for $76.13 \%$. The most OTUs were included in the colonic mucosal tissues of HFD group. According to species accumulation curve (Fig. 1C), when the number of samples reached more than 20, the number of OTUs tended to be stable at 500 , indicating that the $16 \mathrm{~S}$ rDNA sequences in four types of samples (S-HFD for stools of HFD group, S-LFD for stools of LFD group, T-HFD for colon mucosa tissues of HFD group and T-LFD for colon mucosa tissues of 
Table II. Comparison of diversity indices.

\begin{tabular}{lcccc}
\hline Indices & S-HFD $(\mathrm{n}=15)$ & S-LFD $(\mathrm{n}=11)$ & T-HFD $(\mathrm{n}=13)$ & T-LFD $(\mathrm{n}=11)$ \\
\hline Sequences & $30,844 \pm 8,880$ & $29,875 \pm 7,148$ & $31,020 \pm 5,738$ & $29,708 \pm 4,432$ \\
OTUs & $180 \pm 55^{\mathrm{a}}$ & $181 \pm 45$ & $224 \pm 49^{\mathrm{a}}$ & $204 \pm 32$ \\
Observed species & $131.67 \pm 41.73^{\mathrm{a}}$ & $126.91 \pm 36.07^{\mathrm{b}}$ & $168.23 \pm 35.70^{\mathrm{a}}$ & $157.00 \pm 29.25^{\mathrm{b}}$ \\
Shannon & $4.34 \pm 1.03$ & $4.16 \pm 0.85$ & $4.87 \pm 0.56$ & $4.75 \pm 0.48$ \\
Simpson & $0.86 \pm 0.14$ & $0.87 \pm 0.07$ & $0.91 \pm 0.04$ & $0.90 \pm 0.04$ \\
Chao1 & $160.36 \pm 46.20^{\mathrm{a}}$ & $158.15 \pm 36.81^{\mathrm{b}}$ & $203.01 \pm 48.09^{\mathrm{a}}$ & $191.76 \pm 37.11^{\mathrm{b}}$ \\
\hline
\end{tabular}

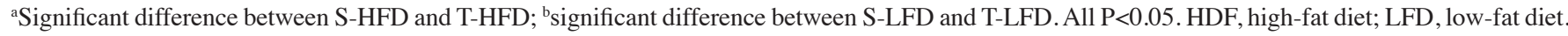
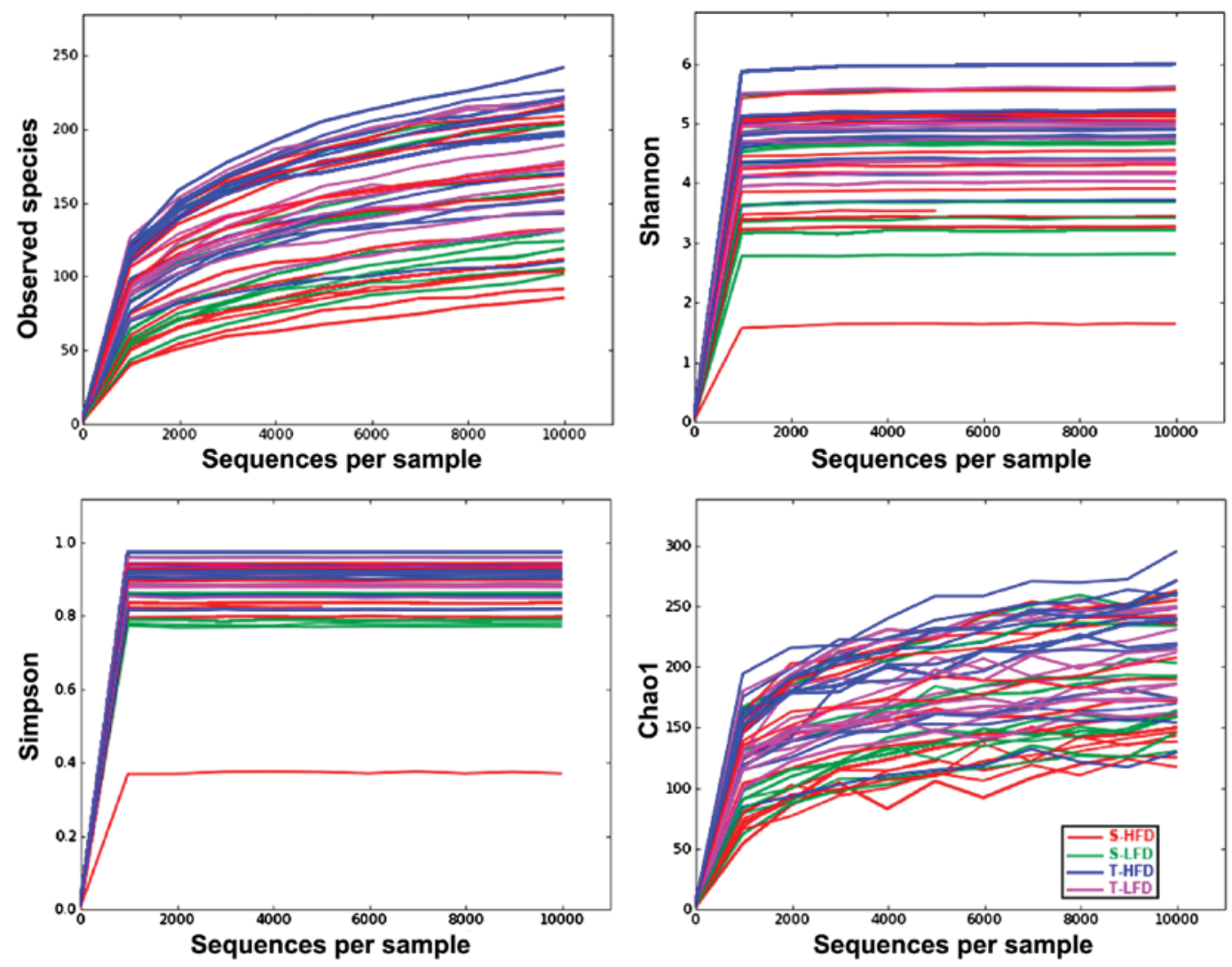

Figure 2. Four $\alpha$ diversity curves of all samples. The color code: Red=S-HFD, green=S-LFD, blue=T-HFD, purple=T-LFD. HDF, high-fat diet; LFD, low-fat diet.

LFD group) represent the majority of bacteria in the samples of this study.

We then analyzed the microbial diversity within each subject ( $\alpha$ diversity) and the difference between each subject's gut microbiota ( $\beta$ diversity). The estimators of $4 \alpha$ diversity indices (Shannon, Chao1, Simpson and observed_species) are shown in Table II. There were no significant differences in these indices between the groups S-HFD and S-LFD, or between the groups T-HFD and T-LFD. However, as shown in Table II, there were statistically significant differences in classification number of OTUs, observed_species and Chao1 indices between the stool samples and colonic mucosal tissue samples in both groups, demonstrating significantly higher diversity found in colonic mucosal tissues compared to stools. Furthermore, according to observed_species and Chaol indices, no rarefaction curve plateaued with the current sequences, suggesting more phylotypes would be expected with additional sequencing (Fig. 2). However, two other indices indicated that most diversity had already been captured. To compare the overall microbiota structure in HFD and LFD subjects, $\beta$ diversity was analyzed using sample clustering and PCoA based on three distance matrices including euclidean, unweighted_uniFrac and weighted_uniFrac (Fig. 3). The results of OTU clustering and PCOA also revealed no significant difference in bacterial structure in stools or colonic mucosal tissues, respectively, but a segregation of stools and colonic mucosal tissues especially on the basis of unweighted UniFrac matrix, as demonstrated by the first three principal component scores which accounted for 15.97, 11.15 and 8.49\% of total variations. In addition, LEfSe was performed to obtain the cladogram representation and the predominant bacteria of the microbiota within stool and colonic mucosal tissue, 
Table III. List of stool and tissue genera significantly different between HFD and LFD groups.

\begin{tabular}{|c|c|c|c|c|c|c|c|c|}
\hline \multirow[b]{2}{*}{ Sample } & \multirow[b]{2}{*}{ Phylum } & \multirow[b]{2}{*}{ Family } & \multirow[b]{2}{*}{ Genus } & \multicolumn{2}{|c|}{$\begin{array}{c}\text { Relative } \\
\text { abundance }(\%)^{\mathrm{a}}\end{array}$} & \multirow[b]{2}{*}{$\kappa^{2}$-value ${ }^{b}$} & \multirow[b]{2}{*}{ P-value } & \multirow{2}{*}{$\begin{array}{c}\text { Tendency in HFD } \\
\text { compared to } \\
\text { LFD group }\end{array}$} \\
\hline & & & & HFD & LFD & & & \\
\hline \multirow[t]{6}{*}{ Stool } & Bacteroidetes & Prevotellaceae & Prevotella & 19.05 & 4.97 & 4.433 & 0.035 & $\uparrow$ \\
\hline & Firmicutes & Aerococcaceae & Abiotrophia & $1.30 \times 10^{-3}$ & 0 & 4.302 & 0.038 & $\uparrow$ \\
\hline & Bacteroidetes & S24-7 & unclassified & 0.19 & 0.29 & 3.965 & 0.046 & $\downarrow$ \\
\hline & Firmicutes & Ruminococcaceae & Gemmiger & 0.11 & 0.52 & 5.616 & 0.018 & $\downarrow$ \\
\hline & Verrucomicrobia & Verrucomicrobiaceae & Akkermansia & 0.03 & 0.19 & 4.447 & 0.035 & $\downarrow$ \\
\hline & Actinobacteria & Micrococcaceae & Rothia & $6.48 \times 10^{-4}$ & $1.63 \times 10^{-3}$ & 4.299 & 0.038 & $\downarrow$ \\
\hline \multirow[t]{5}{*}{ Tissue } & Bacteroidetes & S24-7 & unclassified & 0.1 & 0 & 5.033 & 0.025 & $\uparrow$ \\
\hline & Bacteroidetes & Bacteroidaceae & Bacteroides & 12.96 & 29.03 & 5.237 & 0.022 & $\downarrow$ \\
\hline & Bacteroidetes & Porphyromonadaceae & Coprobacter & 0 & 0.05 & 3.863 & 0.049 & $\downarrow$ \\
\hline & Firmicutes & Aerococcaceae & Abiotrophia & $2.48 \times 10^{-4}$ & 0.02 & 4.387 & 0.036 & $\downarrow$ \\
\hline & Tenericutes & Anaeroplasmataceae & Asteroleplasma & 0 & 0.72 & 3.863 & 0.049 & $\downarrow$ \\
\hline
\end{tabular}

${ }^{a}$ Relative contribution of a genus in a group was calculated as percentage of the sequences of this genus to all sequences in this group. ${ }^{b}$ The $\kappa^{2}$-values were obtained by Mann-Whitney test. HDF, high-fat diet; LFD, low-fat diet.
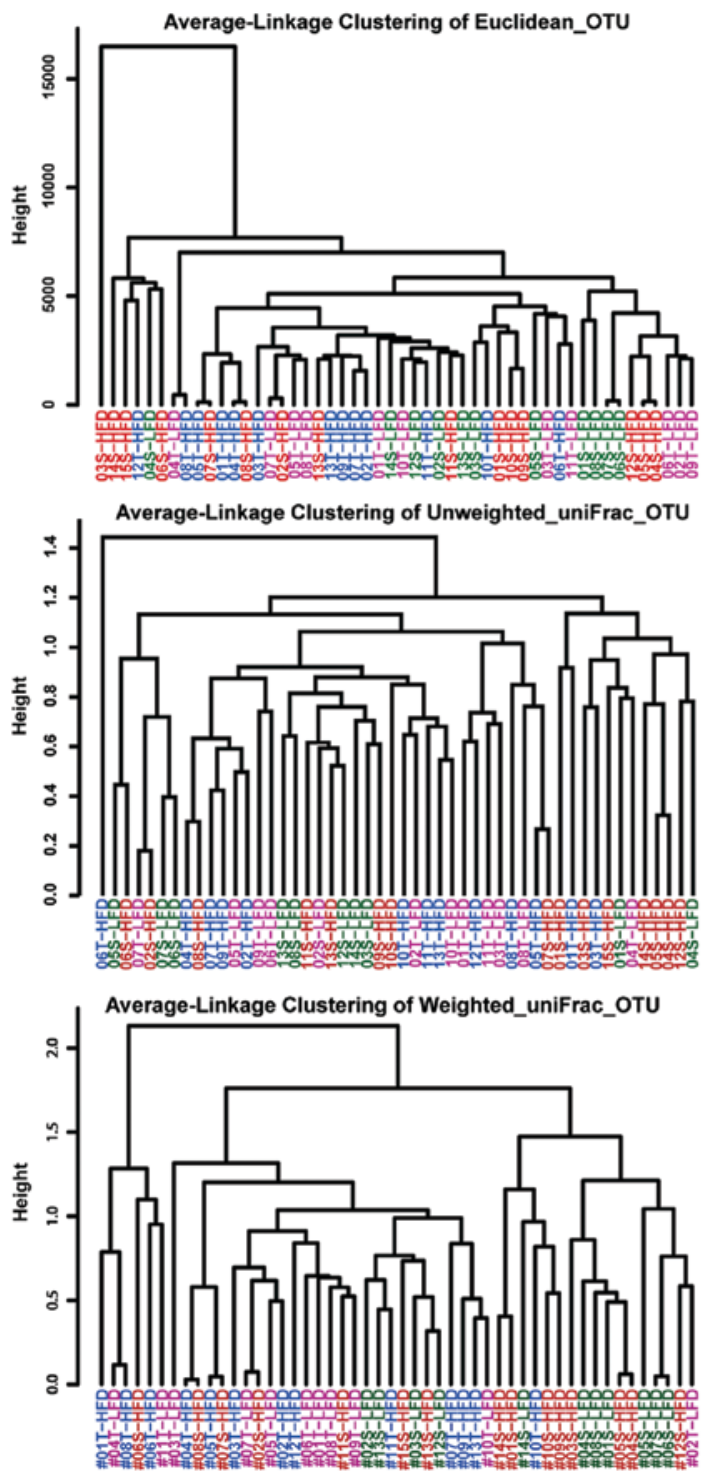

Euclidean_PCoA

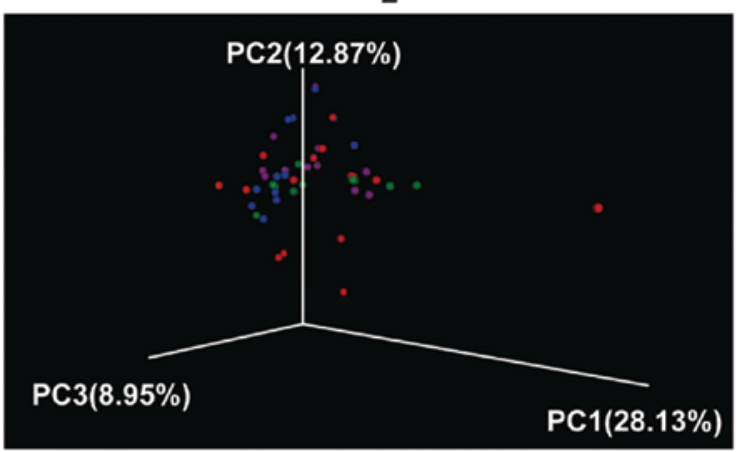

Unweighted_Unifrac_PCoA

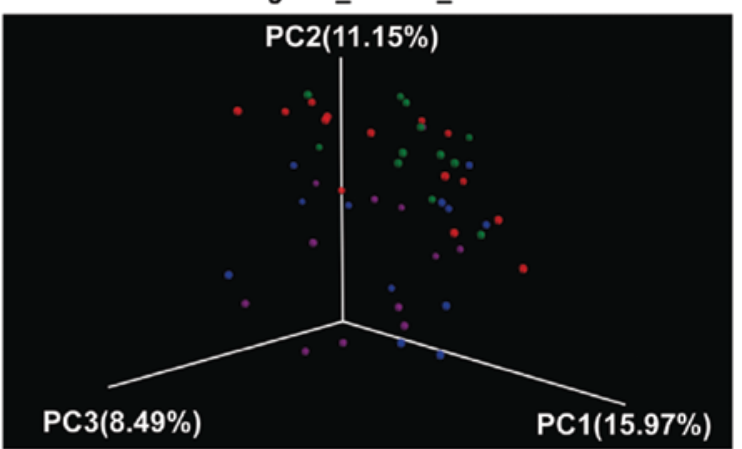

Weighted_uniFrac_PCoA

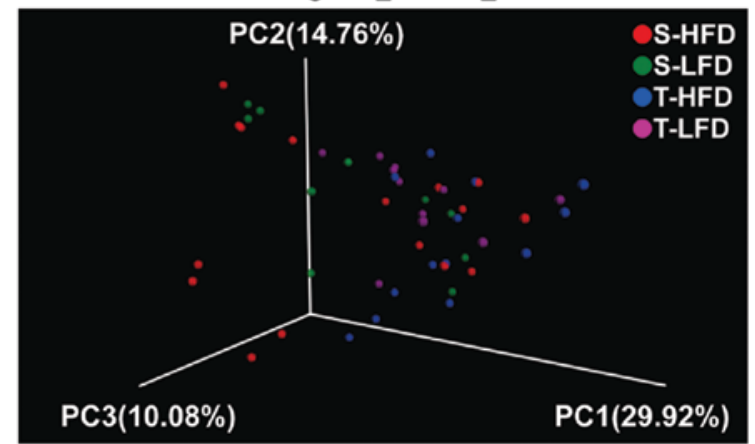

Figure 3. Two $\beta$ diversity plots based on three distance matrices OTU clustering on the left and PCoA on the right. OTU, operational taxonomic unit. 

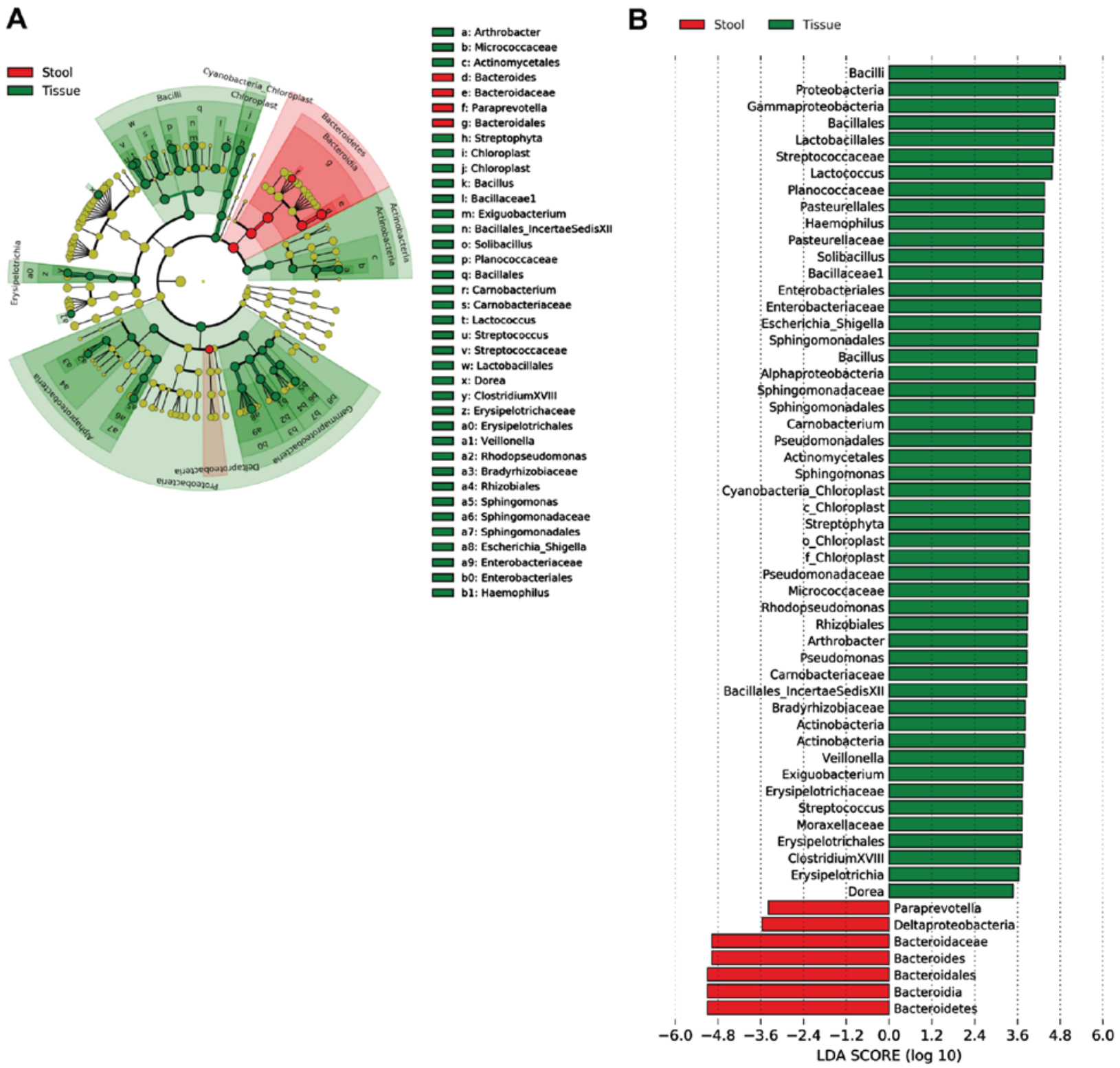

Figure 4. Different structures of microbiota between stool and colon mucosa tissue. (A) Taxonomic representation of statistically and biologically consistent differences between stool and colonic mucosal tissue. Differences are represented by the color of the most abundant class (red indicating stool, green representing colonic mucosal tissue and yellow meaning non-significant). Each circle's diameter is proportional to the taxon's abundance. (B) Histogram of the LDA scores for differential abundance. Cladogram was calculated by LEfSe, and displayed according to effect size.

which is shown in Fig. 4. The differences in taxa between the HFD and LFD groups are depicted in Fig. 5. Paraprevotella was enriched in stool of HFD group, whereas Alistipes and Rikenellaceae were enriched in the stool of LFD group. Bacteroidaceae, Bacteroides and Clostridium XVIII were enriched in colon mucosa tissue of LFD group.

Microbial structures of gut in HFD and LFD volunteers. We analyzed the stool and colonic mucosal tissue bacterial communities of HFD and LFD volunteers, and assessed the amounts of different phyla and genera using taxonomic assignment of all sequences with RDP Classifier. Including all the groups, there were 11 phyla and 129 genera. The overall microbiota structure for each group at the phylum level is shown in Fig. 6, indicating that the tissue bacteria of LFD subjects covered all phyla. The constituent ratios of stool bacteria at phylum level were significantly different from those of tissue bacteria regardless of HFD group or LFD group $(\mathrm{P}<0.001)$, which was consistent with the results of OTU clustering and PCoA.

At the genus level, there were 111 genera in stool microbiota and 117 genera in colonic mucosal tissue microbiota of HFD group, as well as 96 and 117 genera in stool and colonic mucosal tissue microbiota of LFD group, respectively. On comparison of stool genera, HFD group exhibited significantly higher abundance of Prevotella and Abiotrophia and lower abundance of unclassified genus of S24-7 (family level) of Bacteroidetes, Gemmiger, Akkermansia and Rothia, while in colonic mucosa, HFD group showed significantly higher abundance of unclassified genus of S24-7 and a lower abundance of Bacteroides, Coprobacter, Abiotrophia, and Asteroleplasma compared to LFD group (Table III). 
A

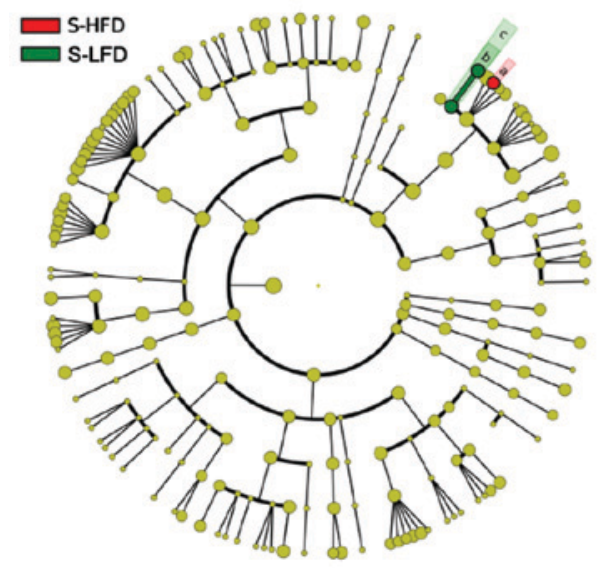

C

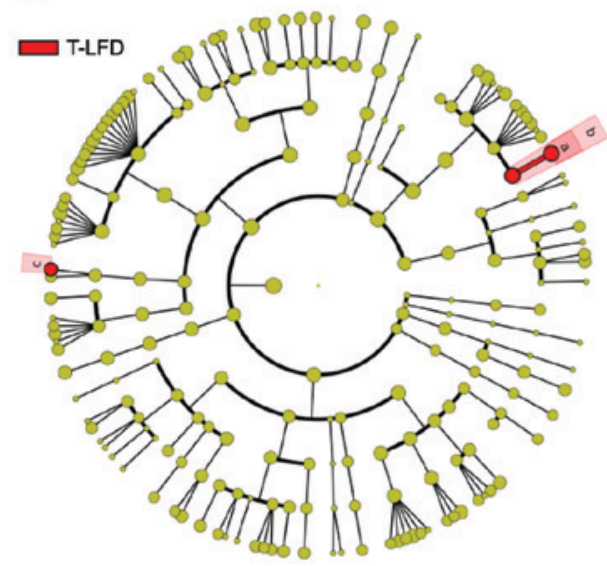

B

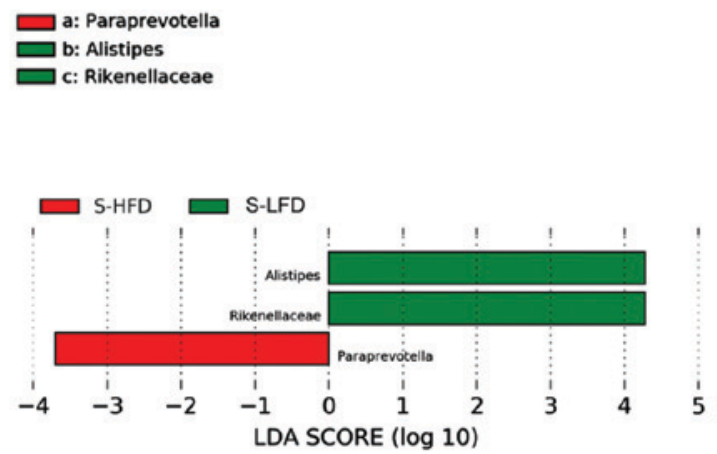

D

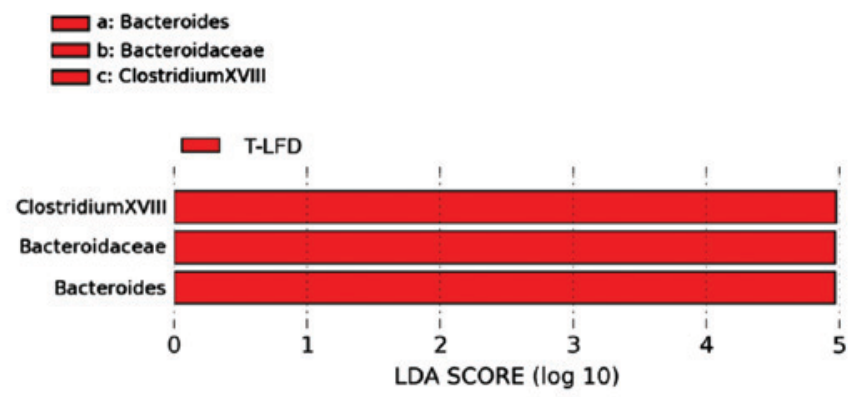

Figure 5. Different structures of stool and colon mucosa tissue microbiota between HFD and LFD groups. (A and C) Taxonomic representation of statistical differences in stool and colonic mucosa between the two groups. (B and D) Histogram of the LDA scores for differentially abundance of taxon. HDF, high-fat diet; LFD, low-fat diet; LDA, linear discriminant analysis.

\section{Discussion}

The findings of the present study prove that diet has a measurable effect on the composition of gut microbiota. Although the concept of enterotypes as robust clustering of human gut community compositions was largely driven by the abundance of key bacterial genera (25), the enterotypes of HFD populations remains to be elucidated. It is well-known that more than $90 \%$ of all the phylotypes at adulthood belong to Bacteroidetes and Firmicutes (26). Each of these enterotypes is identifiable by the variation in the levels of one of three genera: Bacteroides (enterotype 1), Prevotella (enterotype 2) and Ruminococcus (enterotype 3) (27). Wu et al (20) further suggested that microbial enterotypes were significantly affected by long-term diets. They found that the higher intake of proteins and animal fats was associated with higher abundance of the Bacteroides dominant enterotype while the consumption of carbohydraterich diet facilitated growth of the Prevotella enterotype. Kelder et al (28) demonstrated that changes in Bacteroides and Firmicutes levels correlate with changes in carbohydrate and fat oxidation, respectively. Our data showed that Bacteroidetes and Firmicutes together accounted for $80-90 \%$ of the bacterial phyla in stool and colonic mucosal tissue irrespective of the diet composition. However, Bacteroides was the most

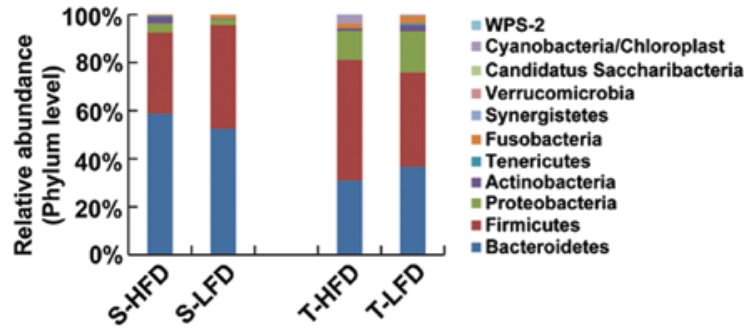

Figure 6. Relative abundance of bacterial phyla in stool and colon mucosa tissue of HFD and LFD groups. Bacteroidetes was the most predominant phylum, contributing 58.76 and $52.42 \%$ of the stool microbiota in HFD and LFD groups, respectively, followed by Firmicutes, which contributed 32.0 and $22.7 \%$, respectively. Proteobacteria, Actinobacteria and Fusobacteria constituted the next most dominant phyla, contributing 3.83 , 2.53 and $0.46 \%$ in HFD group, and $2.38,0.33$ and $1.41 \%$ in LFD group, respectively. The dominant phyla in the tissue microbiota mainly consisted of Firmicutes, Bacteroidetes, Proteobacteria, Cyanobacteria/Chloroplast, Fusobacteria and Actinobacteria, which contributed 50.22, 30.10, 12.06, $3.66,1.94$ and $1.12 \%$ of HFD group, and 39.30, 36.60, 17.13, 0.33, 3.17 and $2.52 \%$ of LFD group, respectively. S, abbreviation for stool; T, abbreviation for colonic mucosal tissue.

abundant phyla in stool, whereas Firmicutes occupied the first phyla in colonic mucosal tissue, which has been rarely reported. The abundance of Proteobacteria in colonic mucosa 
was higher than that in stool. There has been recent interest in the potential associations between gut microbiota and obesity which was thought to be the clinical model of a HFD, in particular, a decreased proportion of Bacteroidetes in obese individuals $(26,29)$. Contrastingly, our study detected a higher abundance of Bacteroidetes in HFD group, most likely as a HFD does not necessarily indicate obesity, or because our probes and primers admittedly do not cover all Bacteroidetes species. Furthermore, due to differences in methodology, results cannot be compared directly to previous $16 \mathrm{~S}$ rDNA based studies. Also, recent work from other researchers could not confirm the proposed association between lower proportions of Bacteroidetes and obesity (30). Combined with a relatively higher proportion of colonic disorders, including colorectitis, polyps, adenomas and carcinoid tumors in HFD group, our observations are consistent with the hypothesis that diet mediated differences in gut microbiota may contribute to the observed increased risk of colorectal diseases in HFD populations $(31,32)$.

It has been previously shown that an increase in the proportion of carbohydrates in the Western diet is remarkably associated with a significantly increased representation of Prevotella $(10,33,34)$. Mozes et al demonstrated, using fluorescent in situ hybridization, that Bacteroides/Prevotella (BAC) prevalence decreased in the jejunum of obese rats consuming HFD (35). Agrarian diets are associated with greater microbial diversity and a predominance of Prevotella over Bacteroides. In contrast, our findings demonstrated that Prevotella constituted more than $19 \%$ of stool microbiota of HFD volunteers. Similarly, vervets consuming a Western diet showed a relatively higher abundance of Prevotella (36). These findings provide the first evidence implying a species-specialization of the gut microbiota. It is also important to note that Western and Asian human HFDs vary markedly across populations. In our study, Chinese high fat diet is mostly related to cooking oil. However, western HFD mostly contains saturated animal fat. The daily intake of fat, protein, and carbohydrate by HFD populations in our study resembled that of the control groups in other studies based on naïve Western and non-Western populations, or even lower, with no difference in the proportions of dietary energy from these three sources between Western and Asian populations (37). Likewise, the data were not generated using the same methodology. Our study further showed that Abiotrophia, which has been associated with infective endocarditis (38), was of a higher representative proportion in HFD populations compared to LFD group. This indicates that a high fat diet may lead to cardiac events through gut dysbiosis. On the contrary, there was significantly lower representation of genera Gemmiger, Akkermansia, and Rothia and an unclassified S24-7 in the feces of HFD subjects. The family S24-7 is a member of Bacteroidetes, belonging to butyrate-producing bacterium, which has been shown to be beneficial for digestive health (39). The decrease of family S24-7 due to a HFD is consistent with findings of other studies (39-41). Some studies suggested that Gemmiger was found to be highly abundant, or stimulated, by prebiotics in the microbiota of subjects with a low BMI suggesting a potential role in weight loss, and positively associated with blood levels of LDL cholesterol and glucose levels $(42,43)$. Akkermansia is a member of the Verrucomicrobia phylum and capable of degrading mucin (44). A growing body of research including rodent and human models of obesity has suggested that Akkermansia spp. abundance in stool negatively correlates with the onset of inflammation, altered adipose tissue metabolism and metabolic syndrome, thus reinforcing the beneficial role of this bacterium on lipid metabolism (45-47). Moreover, recent findings indicated that the higher abundance of Akkermansia muciniphila is also associated with improved glucose metabolism and lower body mass (48), although this has not yet been demonstrated in humans. Rothia is most frequently isolated from the oral cavity and may be associated with periodontal inflammatory disease $(49,50)$. Several studies have indicated that this bacterium may be associated with the occurrence of obesity and obesity-related diseases such as inflammatory bowel disease (IBD) probably by its shift to the gut from the oral cavity $(51,52)$.

The effect of a HFD on the mucosa-associated bacterium community has rarely been reported. Previous studies have shown that the mucosa-associated microbiota differs from the composition of the fecal microbiota, which is in agreement with our study including data of OTU clustering, PCoA and LEfSe analysis. The mucosa-associated microbiota may be particularly important for performing the nutrient exchange at the epithelial interface by converting glycans into shortchain fatty acids (SCFA) that provide energy for the nearby colonocytes and other gut epithelial cells. Such mucosa-associated microbiota are likely to exert a more disproportional effect than the luminal microbiota on the development of the local immune system, and provide resistance against the colonization of pathogenic bacteria at the mucosal epithelial cells in the gut. In this study, we found that five genera had significant differences in prevalence between HFD and LFD groups. Within colonic mucosa, Bacteroides, a member of dominant genera, significantly declined in HFD compared to LFD groups. Most species of Bacteroides scattered among the microbiota-associated phyla degraded the mucin, which possibly led to the availability of oligosaccharides for other bacteria that do not have glycolytic enzymes (53). Several studies have used high-throughput techniques to study the mucosa-associated microbiota in IBD and IBS patients $(54,55)$. Interestingly, there is still no consensus on the changes in Bacteroides abundance in colonic mucosal tissues of intestinal-related diseases (56-58). However, we believe that the decrease of Bacteroides on the mucosal layer due to a HFD may be involved in the perturbation of intestinal mucosal barrier, thereby promoting the occurrence of IBD or $\mathrm{CRC}$. The relative abundance of mucosa-associated unclassified S24-7 increased and genus Abiotrophia decreased in the HFD group, which may explain the inverse prevalence in stool samples. Two genera, Coprobacter and Asteroleplasma, not detectable in the colonic mucosal tissue of HFD populations, were present in small proportions in colonic mucosa of LFD subjects. The genus Coprobacter, first described in 2013, is classified within the family Porphyromonadaceae, the order Bacteroidales, and the phylum Bacteroidetes, and comprise of a single species, Coprobacter fastidiosus $(59,60)$. The genera Asteroleplasmais also rarely reported. The role of these two genera on human health, to date, remains unknown.

In conclusion, gut microbiota is significantly affected by dietary habits and HFD may serve as an etiological factor of colorectal disorders by altering the fecal and mucosal 
microbiota composition especially at genus level. Whether modifying diet or supplementation with protective probiotics can prevent such diseases remains to be elucidated. Furthermore, since colonic diseases such as CRC and the aforementioned illnesses can change the intestinal microbiota composition themselves as shown by fecal transfer studies (17), further investigations are required to link microbiota composition with carcinogenesis.

\section{Acknowledgements}

Not applicable.

\section{Funding}

This study was supported by grants from the National Nature Science Foundation of China (nos. 81472262 and 81230057) and the Emerging Cutting-Edge Technology Joint Research Projects of Shanghai (no. SHDC12012106).

\section{Availability of data and materials}

All data generated or analyzed during this study are included within the article.

\section{Authors' contributions}

LMQ, RYG and HLQ designed the study. LMQ, RYG and JMH collected and analyzed the data. LMH, CP and HL contributed to sample collection and intellectual input. LMQ drafted and wrote the manuscript. RYG and HLQ revised the manuscript critically for intellectual content. All authors gave intellectual input to the study and approved the final version of the manuscript.

\section{Ethics approval and consent to participate}

All procedures performed in studies involving human participants were in accordance with the ethical standards of the Institutional and National Research Committee and with the 1964 Helsinki Declaration and its later amendments or comparable ethical standards. This study was approved by the Ethics Committee of the Institutional Review Boards of Shanghai Tenth People's Hospital (Shanghai, China) and Jiangyin People's Hospital (Jiangyin, China). Signed informed consents were obtained from the patients or the guardians.

\section{Patient consent for publication}

Not applicable.

\section{Competing interests}

The authors declare that they have no competing interests.

\section{References}

1. Chen W, Zheng R, Baade PD, Zhang S, Zeng H, Bray F, Jemal A, Yu XQ and He J: Cancer statistics in China, 2015. CA Cancer J Clin 66: 115-132, 2016.
2. Jobin C: Colorectal cancer: Looking for answers in the microbiota. Cancer Discov 3: 384-387, 2013.

3. Hold GL: Gastrointestinal microbiota and colon cancer. Dig Dis 34: 244-250, 2016

4. Rajilić-Stojanović M, Biagi E, Heilig HG, Kajander K, Kekkonen RA, Tims S and de Vos WM: Global and deep molecular analysis of microbiota signatures in fecal samples from patients with irritable bowel syndrome. Gastroenterology 141: 1792-1801, 2011

5. Ringel Y and Ringel-Kulka T: The Intestinal microbiota and irritable bowel syndrome. J Clin Gastroenterol 49 (Suppl 1): S56-S59, 2015.

6. Sepehri S, Kotlowski R, Bernstein CN and Krause DO: Microbial diversity of inflamed and noninflamed gut biopsy tissues in inflammatory bowel disease. Inflamm Bowel Dis 13: 675-683, 2007.

7. Chang $\mathrm{C}$ and Lin H: Dysbiosis in gastrointestinal disorders. Best Pract Res Clin Gastroenterol 30: 3-15, 2016.

8. Bassaganya-Riera J, Viladomiu M, Pedragosa M, De Simone C and Hontecillas R: Immunoregulatory mechanisms underlying prevention of colitis-associated colorectal cancer by probiotic bacteria. PLoS One 7: e34676, 2012.

9. Fukugaiti MH, Ignacio A, Fernandes MR, Ribeiro Júnior U, Nakano V and Avila-Campos MJ: High occurrence of Fusobacterium nucleatum and Clostridium difficile in the intestinal microbiota of colorectal carcinoma patients. Braz J Microbiol 46: 1135-1140, 2015

10. Conlon MA and Bird AR: The impact of diet and lifestyle on gut microbiota and human health. Nutrients 7: 17-44, 2014.

11. Ridlon JM, Kang DJ and Hylemon PB: Bile salt biotransformations by human intestinal bacteria. J Lipid Res 47: 241-259, 2006.

12. Ou J, DeLany JP, Zhang M, Sharma S and O'Keefe SJ: Association between low colonic short-chain fatty acids and high bile acids in high colon cancer risk populations. Nutr Cancer 64: 34-40, 2012.

13. Nicholson JK, Holmes E, Kinross J, Burcelin R, Gibson G, Jia W and Pettersson S: Host-gut microbiota metabolic interactions. Science 336: 1262-1267, 2012.

14. Shen CL, Purewal M, San Francisco S and Pence BC: Absence of PhIP adducts, p53 and Apc mutations, in rats fed a cooked beef diet containing a high level of heterocyclic amines. Nutr Cancer 30: 227-231, 1998 .

15. Butler LM, Sinha R, Millikan RC, Martin CF, Newman B, Gammon MD, Ammerman AS and Sandler RS: Heterocyclic amines, meat intake, and association with colon cancer in a population-based study. Am J Epidemiol 157: 434-445, 2003.

16. Louis P, Hold GL and Flint HJ: The gut microbiota, bacterial metabolites and colorectal cancer. Nat Rev Microbiol 12: 661-672, 2014.

17. Schulz MD, Atay C, Heringer J, Romrig FK, Schwitalla S, Aydin B, Ziegler PK, Varga J, Reindl W, Pommerenke C, et al: High-fat-diet-mediated dysbiosis promotes intestinal carcinogenesis independently of obesity. Nature 514: 508-512, 2014.

18. Yu YN and Fang JY: Gut microbiota and colorectal cancer. Gastrointest Tumors 2: 26-32, 2015.

19. Turnbaugh PJ, Ridaura VK, Faith JJ, Rey FE, Knight R and Gordon JI: The effect of diet on the human gut microbiome: A metagenomic analysis in humanized gnotobiotic mice. Sci Transl Med 1: 6ra14, 2009.

20. Wu GD, Chen J,Hoffmann C, Bittinger K, Chen YY, Keilbaugh SA, Bewtra M, Knights D, Walters WA, Knight R, et al: Linking long-term dietary patterns with gut microbial enterotypes. Science 334: 105-108, 2011

21. Williams T: Building health information systems in the context of national strategies for the development of statistics. Bull World Health Organ 83: 564, 2005.

22. Zhao W, Hasegawa $\mathrm{K}$ and Chen J: The use of food-frequency questionnaires for various purposes in China. Public Health Nutr 5 (6A): 829-833, 2002

23. Chiu BC, Ji BT, Dai Q, Gridley G, McLaughlin JK, Gao YT, Fraumeni JF Jr and Chow WH: Dietary factors and risk of colon cancer in Shanghai, China. Cancer Epidemiol Biomarkers Prev 12: 201-208, 2003

24. Li W and Godzik A: Cd-hit: A fast program for clustering and comparing large sets of protein or nucleotide sequences. Bioinformatics 22: 1658-1659, 2006.

25. Koren O, Knights D, Gonzalez A, Waldron L, Segata N, Knight R, Huttenhower C and Ley RE: A guide to enterotypes across the human body: Meta-analysis of microbial community structures in human microbiome datasets. PLOS Comput Biol 9: e1002863, 2013. 
26. Ley RE, Turnbaugh PJ, Klein S and Gordon JI: Microbial ecology: Human gut microbes associated with obesity. Nature 444: 1022-1023, 2006.

27. Arumugam M, Raes J, Pelletier E, Le Paslier D, Yamada T, Mende DR, Fernandes GR, Tap J, Bruls T, Batto JM, et al MetaHIT Consortium: Enterotypes of the human gut microbiome. Nature 473: 174-180, 2011.

28. Kelder T, Stroeve JH, Bijlsma S, Radonjic M and Roeselers G: Correlation network analysis reveals relationships between diet-induced changes in human gut microbiota and metabolic health. Nutr Diabetes 4: e122, 2014.

29. Ley RE, Bäckhed F, Turnbaugh P, Lozupone CA, Knight RD and Gordon JI: Obesity alters gut microbial ecology. Proc Natl Acad Sci USA 102: 11070-11075, 2005.

30. Duncan SH, Lobley GE, Holtrop G, Ince J, Johnstone AM, Louis $\mathrm{P}$ and Flint HJ: Human colonic microbiota associated with diet, obesity and weight loss. Int J Obes 32: 1720-1724, 2008.

31. Vipperla K and O'Keefe SJ: Diet, microbiota, and dysbiosis: A 'recipe' for colorectal cancer. Food Funct 7: 1731-1740, 2016.

32. Brown K, DeCoffe D, Molcan E and Gibson DL: Diet-induced dysbiosis of the intestinal microbiota and the effects on immunity and disease. Nutrients 4: 1095-1119, 2012.

33. Bleau C, Karelis AD, St-Pierre DH and Lamontagne L: Crosstalk between intestinal microbiota, adipose tissue and skeletal muscle as an early event in systemic low-grade inflammation and the development of obesity and diabetes. Diabetes Metab Res Rev 31: 545-561, 2015

34. Kovatcheva-Datchary P, Nilsson A, Akrami R, Lee YS, De Vadder F, Arora T, Hallen A, Martens E, Björck I and Bäckhed F: Dietary fiber-induced improvement in glucose metabolism is associated with increased abundance of Prevotella. Cell Metab 22: 971-982, 2015.

35. Mozes S, Bujnáková D, Sefcíková Z and Kmet V: Developmental changes of gut microflora and enzyme activity in rat pups exposed to fat-rich diet. Obesity (Silver Spring) 16: 2610-2615, 2008.

36. Amato KR, Yeoman CJ, Cerda G, Schmitt CA, Cramer JD, Miller ME, Gomez A, Turner TR, Wilson BA, Stumpf RM, et al: Variable responses of human and non-human primate gut microbiomes to a Western diet. Microbiome 3: 53, 2015.

37. Ou J, Carbonero F, Zoetendal EG, DeLany JP, Wang M, Newton K, Gaskins HR and O'Keefe SJ: Diet, microbiota, and microbial metabolites in colon cancer risk in rural Africans and African Americans. Am J Clin Nutr 98: 111-120, 2013.

38. Al-Jasser AM, Enani MA and Al-Fagih MR: Endocarditis caused by Abiotrophia defectiva. Libyan J Med 2: 43-45, 2007.

39. Evans CC, LePard KJ, Kwak JW, Stancukas MC, Laskowski S Dougherty J, Moulton L, Glawe A, Wang Y, Leone V, et al: Exercise prevents weight gain and alters the gut microbiota in a mouse model of high fat diet-induced obesity. PLoS One 9: e92193, 2014.

40. Pyndt Jørgensen B, Hansen JT, Krych L, Larsen C, Klein AB, Nielsen DS, Josefsen K, Hansen AK and Sørensen DB: A possible link between food and mood: Dietary impact on gut microbiota and behavior in BALB/c mice. PLoS One 9: e103398, 2014.

41. Tomas J, Mulet C, Saffarian A, Cavin JB, Ducroc R, Regnault B, Kun Tan C, Duszka K, Burcelin R, Wahli W, et al: High-fat diet modifies the PPAR- $\gamma$ pathway leading to disruption of microbial and physiological ecosystem in murine small intestine. Proc Nat Acad Sci USA 113: E5934-E5943, 2016

42. Lagkouvardos I, Kläring K, Heinzmann SS, Platz S, Scholz B, Engel KH, Schmitt-Kopplin P, Haller D, Rohn S, Skurk T, et al: Gut metabolites and bacterial community networks during a pilot intervention study with flaxseeds in healthy adult men. Mol Nutr Food Res 59: 1614-1628, 2015.

43. Patrone V, Vajana E, Minuti A, Callegari ML, Federico A, Loguercio C, Dallio M, Tolone S, Docimo L and Morelli L: Postoperative changes in fecal bacterial communities and fermentation products in obese patients undergoing bilio-intestinal bypass. Front Microbiol 7: 200, 2016.

44. Everard A, Belzer C, Geurts L, Ouwerkerk JP, Druart C, Bindels LB, Guiot Y,Derrien M,Muccioli GG,DelzenneNM, et al: Cross-talk between Akkermansia muciniphila and intestinal epithelium controls diet-induced obesity. Proc Natl Acad Sci USA 110: 9066-9071, 2013.
45. Anhê FF, Roy D, Pilon G, Dudonné S, Matamoros S, Varin TV, Garofalo C, Moine Q, Desjardins Y, Levy E, et al: A polyphenolrich cranberry extract protects from diet-induced obesity, insulin resistance and intestinal inflammation in association with increased Akkermansia spp. population in the gut microbiota of mice. Gut 64: 872-883, 2015.

46. Schneeberger M, Everard A, Gómez-Valadés AG, Matamoros S, Ramírez S, Delzenne NM, Gomis R, Claret M and Cani PD: Akkermansia muciniphila inversely correlates with the onset of inflammation, altered adipose tissue metabolism and metabolic disorders during obesity in mice. Sci Rep 5: 16643, 2015.

47. Shin NR, Lee JC, Lee HY, Kim MS, Whon TW, Lee MS and Bae JW: An increase in the Akkermansia spp. population induced by metformin treatment improves glucose homeostasis in diet-induced obese mice. Gut 63: 727-735, 2014.

48. Harakeh SM, Khan I, Kumosani T, Barbour E, Almasaudi SB, Bahijri SM, Alfadul SM, Ajabnoor GM and Azhar EI: Gut microbiota: A contributing factor to obesity. Front Cell Infect Microbiol 6: 95, 2016

49. Huang S, Li R, Zeng X, He T, Zhao H, Chang A, Bo C, Chen J, Yang F, Knight R, et al: Predictive modeling of gingivitis severity and susceptibility via oral microbiota. ISME J 8: 1768-1780, 2014

50. Kataoka H, Taniguchi M, Fukamachi H, Arimoto T, Morisaki H and Kuwata H: Rothia dentocariosa induces TNF-alpha production in a TLR2-dependent manner. Pathog Dis 71: 65-68, 2014.

51. Verma M, Hontecillas R, Abedi V, Leber A, Tubau-Juni N, Philipson C, Carbo A and Bassaganya-Riera J: Modeling-enabled systems nutritional immunology. Front Nutr 3: 5, 2016.

52. Yasir M, Angelakis E, Bibi F, Azhar EI, Bachar D, Lagier JC, Gaborit B, Hassan AM, Jiman-Fatani AA, Alshali KZ, et al: Comparison of the gut microbiota of people in France and Saudi Arabia. Nutr Diabetes 5: e153, 2015.

53. Lee SM, Donaldson GP, Mikulski Z, Boyajian S, Ley K and Mazmanian SK: Bacterial colonization factors control specificity and stability of the gut microbiota. Nature 501: 426-429, 2013.

54. Walker AW, Sanderson JD, Churcher C, Parkes GC, Hudspith BN, Rayment N, Brostoff J, Parkhill J, Dougan G and Petrovska L: High-throughput clone library analysis of the mucosa-associated microbiota reveals dysbiosis and differences between inflamed and non-inflamed regions of the intestine in inflammatory bowel disease. BMC Microbiol 11: 7, 2011.

55. Parkes GC, Rayment NB, Hudspith BN, Petrovska L, Lomer MC, Brostoff J, Whelan K and Sanderson JD: Distinct microbial populations exist in the mucosa-associated microbiota of sub-groups of irritable bowel syndrome. Neurogastroenterol Motil 24: 31-39, 2012.

56. Verma R, Verma AK, Ahuja V and Paul J: Real-time analysis of mucosal flora in patients with inflammatory bowel disease in India. J Clin Microbiol 48: 4279-4282, 2010.

57. Hans W, Schölmerich J, Gross V and Falk W: The role of the resident intestinal flora in acute and chronic dextran sulfate sodium-induced colitis in mice. Eur J Gastroenterol Hepatol 12: 267-273, 2000

58. Yao P, Cui M, Wang H, Gao H, Wang L, Yang T and Cheng Y: Quantitative analysis of intestinal flora of Uygur and Han Ethnic Chinese patients with ulcerative colitis. Gastroenterol Res Pract 2016: 9186232, 2016.

59. Chaplin AV, Efimov BA, Khokhlova EV, Kafarskaia LI, Tupikin AE, Kabilov MR and Shkoporov AN: Draft genome sequence of Coprobacter fastidiosus NSB1T. Genome Announc 2: 2, 2014.

60. Nakata T, Kyoui D, Takahashi H,Kimura B and Kuda T: Inhibitory effects of laminaran and alginate on production of putrefactive compounds from soy protein by intestinal microbiota in vitro and in rats. Carbohydr Polym 143: 61-69, 2016.

This work is licensed under a Creative Commons Attribution-NonCommercial-NoDerivatives 4.0 International (CC BY-NC-ND 4.0) License. 